\title{
Mini Gesture Detection Using Neural Networks Algorithms
}

\author{
Norah Alnaim ${ }^{1}$, and Maysam Abbod ${ }^{2}$ \\ ${ }^{1,2}$ Department of Electronic and Computer Engineering, Brunel University London, London, UK \\ Norah.Alnaim@brunel.ac.uk \\ Maysam.Abbod@brunel.ac.uk
}

\begin{abstract}
Gesture recognition is defined as non-verbal motions used as a means of communication in Human Computer Interaction. It is one of the significant aspects of HCI, both in the device interfaces and interpersonally. In a virtual reality system, gestures can be used to navigate, control or interact with a computer. The aim of gesture recognition is to capture gestures that are formed in a certain way and are detected by a device such as a camera. Hand gesture recognition is one of the logical ways to generate a convenient and high adaptability interface between devices and users. In this paper, a system is created for hand gesture recognition using image processing tools, namely Wavelets Transform (WT), Empirical Mode Decomposition (EMD) methods, Artificial Neural Networks (ANN) and Convolutional Neural Network $(\mathrm{CNN})$, for gesture classification. These methods are evaluated based on many factors such as execution time, accuracy, sensitivity, specificity, positive and negative predictive value, likelihood, receiver operating characteristic, area under roc curve and root mean square. Preliminary results indicate that WT had less execution time than EMD and CNN. CNN had the ability to extract distinct features and classify data accurately while EMD and WT were less effective. Hence, the classification accuracy is improved dramatically.
\end{abstract}

Keywords: Wavelet Transform, Empirical Mode Decomposition, Artificial Neural Networks, Convolutional Neural Network, Hand Gesture Recognition, HCI.

\section{INTRODUCTION}

The hand is often well-known as the most natural and instinctive interaction for humans. People often tend to communicate signals and messages non- verbally using hand gestures. Sign languages have been the only way to communicate with deaf people for a long time. In Human-Computer Interaction (HCI) world, an appropriate hand tracking is a tracking phase which helps to develop instinctive HCI system that can be used in applications akin to virtual object manipulation, gaming and gesture recognition. Moreover, hand tracking is an interesting principle point which deals with three main parts of computer vision that are hand segmentation, detection, and tracking. The hand gesture is one of the expressive way used in healthcare, education and the entertainment industry too that could be used by special needs people and elders who become partly incapable of movements.

Hand motion could be detected using any type of camera that offers reasonable image quality. 2-D cameras can easily be used in detecting most hand motions on a constant surface such as Microsoft that designed a depth camera with particular motion sensing named Kinect. Alternatively, Intel produced a small Interactive gesture camera that has reasonable specifications. Apple is also one of the leaders in gesture recognition worldwide by designing the latest versions of the iPhone with a small high-quality camera. The video recording is up to $4 \mathrm{k}$ at 24,30 and 60 frames per second (fps).

It is known that the video contains a considerable number of images that are connected together to form a clip. An image will down sample into a number of frames thus each frame gets into several stages for image processing. The functionality of all image processing techniques is similar with few differences in each phase. Image processing phases consist of five phases, which are data input, pre-processing, image segmentation, feature extraction, and classification.

There are many detection methods available for hand gestures. In this study, a system is created for hand gesture recognition using the following image processing tools, namely Wavelets Transform (WT), Empirical Mode Decomposition (EMD) methods, Artificial Neural Networks (ANN) and Convolutional Neural Network (CNN) for gesture classification. These methods are evaluated based on execution time, accuracy, sensitivity, specificity, positive predictive value, negative predictive value, positive likelihood, negative likelihood, receiver operating characteristic, area under roc curve and root mean square. WT and EMD methods will be employed in two dimensions. For the 
classification, ANN will be used to classify the gestures using the features extracted as inputs to the ANN. Multiple training sessions would be done where filters would be applied. Each output of one stage will be the input of the next one. All previous stages will be shortened by using CNN deep learning tool. The objective of the study is to identify the best method available to extract features when the classification accuracy is being compared using WT, EMD, and CNN as deep learning techniques.

\section{LITERATURE REVIEW}

To extract some useful information or enhancing the image, some operations are performed on images. The methods of performing these operations on images are called image processing. Just like signal processing, the input is an image while the output may be an enhanced image or some attribute related to the input image. Two types of techniques are used for two types of image. Analog image processing targets analogue image photographs, while digital image processing emphasizes on digital images. Since the focus of this document is mainly based on digital image processing, we will restrain this discussion to digital image processing. The digital image processing can be defined as processing an array of real numbers presented by a number of bits. The field of image processing plays a vital role and contribute towards the solution of many problems, which includes security, remote sensing applications, industries, medical etc. [1].

Image segmentation is one of the important part of image processing techniques. Segmentation is the process of distinguishing the image in various ways, depending on the nature of the problem. Several researchers propose their work on image segmentation from various domains. Since there is no solution to the image segmentation problem, domain knowledge is combined with the techniques that belongs to the solution of different domains. [2] [3].

Feature detection is a crucial part of 2-D and 3-D image processing [4]. Before any feature extraction technique is applied, the image data is pre-processed, and different pre-processing techniques are applied to images such as thresholding, binarization, and normalization, etc. Features are then extracted and used for classification purposes. Features of an image present the behaviour of an image. A preferable feature set contains attributes with high information gain and can provide positive outcome of classification of images into different classes.

Extraction and classification of local image structure are discussed by Gevers et al. [5] in their research paper. For most of the image processing and computer vision tasks, object recognition, stereo vision and 3-D reconstruction extraction and classification of local image structure are very important. Using the geometrical and photometrical information, they proposed a method which classifies the physical nature of local image structure.

Murthy and Jadon [6] proposed a new method in Hand Gesture Recognition using Neural Networks. They designed a system that could recognize hand gesture and use these gestures to send information. Any user could display his/her hand and do a particular gesture in front of the web camera that is connected to a computer at any time. Supervised feedforward neural network net-based training and back-propagation technique to classify hand gesture in ten various categories: hand pointing up, down, left, right and front, and also a number of fingers user was showing in the result. The results might reach up to $89 \%$ correct results on the test set.

Pradipa and Kavitha [7] presented a survey paper on the technique used in gesture recognition. According to them, the main aim of gesture recognition is by developing a system which can detect human actions and use them to extract meaningful information for device control. In the case where human-computer interaction hand gesture can play a significant role where people's verbal disabilities can take full advantages of computer systems. This will also help in reducing the use of hardware devices, the use of operating the computer system and causes less greenhouse gas emitting.

Manresa et al., [8] proposed an algorithm for tracking and recognizing hand gestures to interact with video games. This real-time algorithm has three steps. The first step is the hand segmentation in which they use colour values of human skin due to its irregular properties and computational simplicity. The second step is to overcome the errors due to the segmentation process and named it as tracking. Constant velocity model and pixel labelling approach are used to perform tracking. The third step is gesture recognition. Several different hand features are extracted during second steps and finite state classifier is used in identifying the hand configuration. There are four gesture classes, and the classifier is designed to classify the hand gesture in one of the classes. The proposed algorithm is demonstrated in a controlled video game environment.

Pang et al., [9] presented a new method to understand visual tracking target tasks. This proposed technique is an application of deep learning, heuristic, and transfer learning, and uses a convolutional neural network tool, which is able to arrange a set of areas based on how to fit the target is framed. 36 different algorithms on 50 videos were performed. 
Tahani Bouchrika et al. [10] work output is based upon making amendments in the Wavelet Network classification phase of the algorithm by making separated Wavelet Networks discriminating classes $(n-1)$ with the purpose of training each image which results in less time required to complete the testing phase. The proposed Wavelet Network has new architecture composed of stages that learns quickly and recognizes actions by avoiding unnecessary hand movements.

Bouchrika et al. [11] worked with still and continuous moving hand gestures present in a video. Their aim was to command computer system through gesture recognition. They used wavelet network classifier along with neural network classifier for comparison.

\section{THEORY}

\subsection{Wavelets Transforms}

Wavelet transforms are one of the image processing algorithms performing the signal analysis where signal frequency differs at the end of time [12]. Wavelet transforms analysis offers accurate information with regards to signal data in comparison to other analysis techniques. The type of wavelet used in this experiment is the Daubechies orthogonal which is known as dbN wavelets where $\mathrm{N}$ is the number of fading moments. The application is commonly used for audio, speech, image, video, and bio-medical Imaging. The Daubechies wavelets are defined as follows:

$$
\int x^{n} \psi(x) d x=0, \quad n=0,1, \cdots, K
$$

The equation has a combination of scaling functions that is used to represent numerical approximations on a secured scale. The value of $\mathrm{K}$ is directly proportional to the orthogonality condition.

\subsection{Empirical Mode Decomposition}

EMD is an innovative technology used in both non-stationary and non-linear data [13]. The functionality of this method is based on decomposing a signal into Intrinsic Mode Functions (IMF) with respect to the time domain [14] [15]. EMD method could be compared to other analysis techniques such as Wavelet Transforms and Fourier Transforms [15]. EMD technology might be applied to data related to seismic readings, results of neuroscience experiments, electrocardiograms, gastroelectrograms, and sea-surface height readings [16] [17]. The EMD is defined as follows:

$$
x(t)=\sum_{n=1}^{N} c_{n}(t)+r_{n}(t)
$$

Where $r_{n}$ is the mean trend of $x(t)$, the value of $c_{n}$ are the of amplitude and frequency modulated output set. The frequency decreases as the value of $c_{n}$ increases.

\subsection{Artificial Neural Networks}

ANN is simple electronic model similar to the neural structure of the human brain. The functionality of brain learns from the experience around us [16] [17]. ANN is a system that processes information which has the similar functionality of the biological nervous system. The key aspect of this system is the unique structure of the information processing system [16] [17]. The system is composed of enormous number of unified processing elements working together to solve certain issues [16] [17]. It is specifically configured for data classification or pattern recognition application via learning process [16] [17]. There are several benefits associated with using ANN such as self-learning and large data handling.

\subsection{Convolutional Neural Networks}

CNN is a different type of multi-layer neural network and each layer of a CNN converts one amount of activations to another through a function. CNN is a special architecture used for deep learning [9]. To build CNN architecture, it applies three key types of layer: Convolutional Layer, Pooling Layer, and Fully-Connected Layer. It is trained with a type of the back-propagation system which differs in the architecture. CNN is frequently used in recognizing scenes and objects, and carry out image detection, extraction and segmentation. Due to three factors which removes the necessity for feature extraction by using image processing tools, the image data is learned directly by CNN; CNN makes a unique art for recognition results and it might be retrained easily for new recognition missions; CNN is allowed to build on the preexisting network. All the following factors have made the usage of CNN significant in last few years [9]. 


\section{SYSTEM IMPLEMENTATIONS}

\subsection{Hand Gestures Input}

In this study, hand gestures are the input to different gesture detection algorithms. Figure 1 shows 10 various hand gestures which are recorded in short distance with a plain background that is used in this experiment. Some motions are 2-D while others are 3-D.

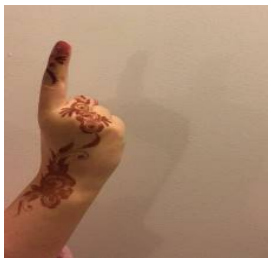

a) Sweep motion

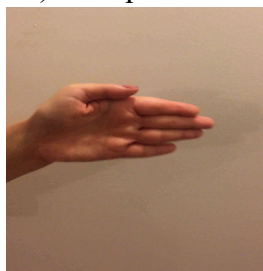

f) Back/Forth

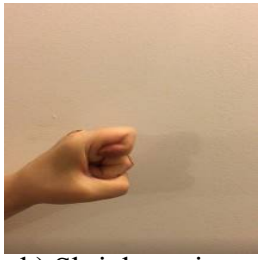

b) Shrink motion

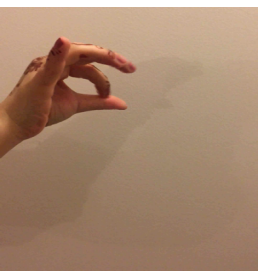

g) Rub motion
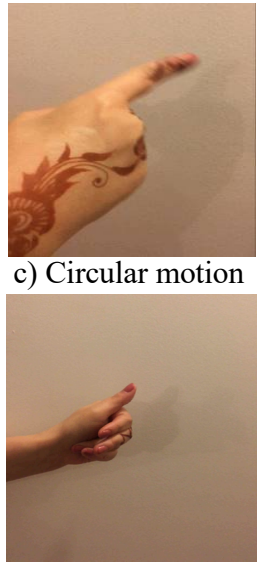

h) Click motion

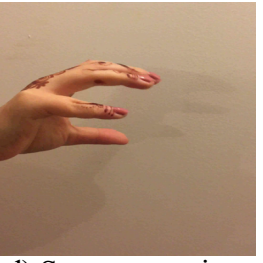

d) Squeeze motion

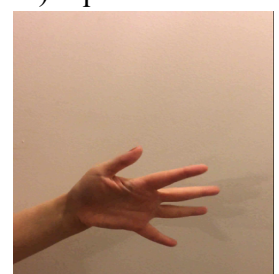

i) Dance motion

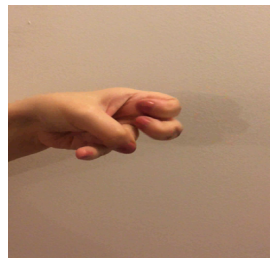

e) 2 Fingers Shrink

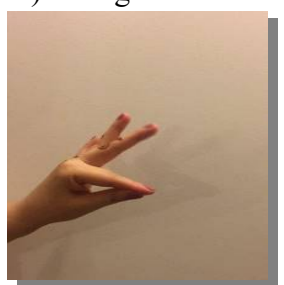

j) Pinch motion

Figure 1. Different hand gestures

Figure 2 shows the framework of implementation, explained in the next steps, used to extract and classify hand motions precisely. By using iPhone 6 Plus camera resolution $4 \mathrm{k}$ at $30 \mathrm{fps}$, ten different hand motions in short distance are recorded with a plain background. The dataset is the author's hand and it uploaded directly to the PC to prepare them for multi-processes. Each recording lasts 10 seconds and the resolution of the recorded video is $3840 \times 2160$. The first system is created using optical flow object by estimating and displaying the optical flow of objects in the video. The length of videos is between 15 to 65 frames. Each video has a different number of frames, which depends on the first section of motion.

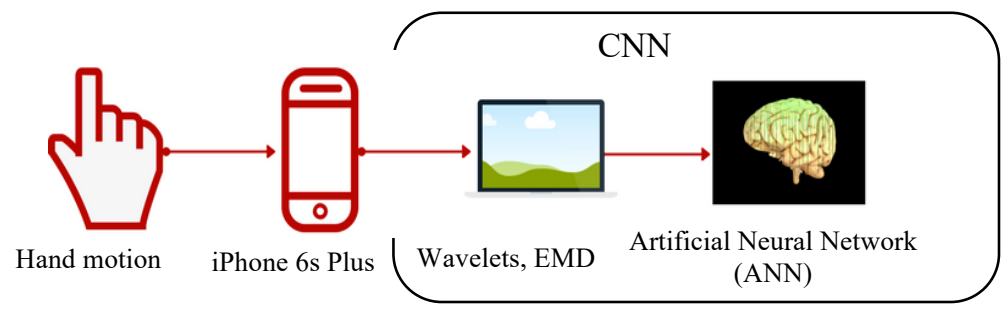

Figure 2. Illustrated framework of system implementation

\subsection{Computing Platform Specification}

The experiment was performed using a Dell laptop XPS 159550 with $6^{\text {th }}$ processor Generation Intel Quad Core i7, memory type DDR4 16 GB speed $2133 \mathrm{MHz}, 512 \mathrm{~GB}$ storage hard drive, 15.6-inch Ultra-HD 15.6" IPS 1920×1080 RGB Optional $3840 \times 2160$ IGZO IPS display w/Adobe RGB colour space and touch. Operating system platform is incorporated within windows 10 of 64 bits. The system is implemented using MATLAB R2017bV language.

\subsection{Implement Wavelet Transform with Artificial Neural Network}

The system is implemented using the db8 WT tool into the following stages:

1. Read each video using video reader function

2. Create optical flow object that spreads the object velocities in an image.

3. Estimate and display the optical flow of objects in the video. 
4. Divide a video into certain frames; each frame contains 8 IMFs.

5. Apply appcoef 2 function which is used to compute an approximation coefficient of 2-D signals.

6. Extract each level using wrcoef function to reconstruct the coefficients of each level in the video.

7. The execution time of WT is estimated only once.

8. The image data is trained and tested using a Neural Network system. The NN has 9 hidden layers to train data in each layer and it stops when the error is reached in 20 epochs. When we add more hidden layers and increase the depth of the neural network, the neural network model becomes a deep learning model. Thus, 9 layers are selected for this experiment.

9. The execution time of image data training and testing are also calculated.

\subsection{Empirical Mode Decomposition with Neural Network Implementation}

The implementation of EMD is similar to WT with some variances. The same 4 steps of WT are used, but with different functions: reshape function (returns the M-by-N matrix whose elements takes column-wise from X, ceemd function (a noise improved data analysis algorithm) complementary collaborates with EMD.

\subsection{Convolutional Neural Network Implementation}

Deep learning has an intelligent method such as CNN, which is used to train data without requiring any image processing tool. In our experiment, we made a new directory for each video. 10 images are generated to transfer the image frame RGB to grey and resize it to $48 \times 27$. All videos have 70 frames. The image's data is split into training and testing datasets. The CNN topology is created in 7 layers; each layer has the following functionality and size: ImageInputLayer Input size [48,27,1], Convolution2DLayer Filter size [5,5], ReLULayer (Rectified Linear Unit), MaxPooling2DLayer Pool size [2,2], FullyConnectedLayer Input size [auto] and Output size [12], SoftmaxLayer and ClassificationOutputLayer Output size [auto]. The hyperparameters of the CNN is generated inside training options function. The value of max epochs parameter is set to 200 epochs.

\subsection{Parameters for Comparison}

In this study, the WT, EMD and CCN algorithms will be compared based upon the following explained parameters. Execution time is the processing amount of duration taken by the software to process the given task. Sensitivity simply measures the percentage of positives which are properly identified. Specificity is a measure of the false positive rate. The PPV and NPV are the percentages of positive and negative results in diagnostic and statistics tests which also described the true positive and true negative results. The LR+ and LR- are one of known measures in diagnostic accuracy. Area under ROC curve (AUC) is the typical technique to measure the accuracy of predictive models.

\section{RESULTS}

The system is implemented ten times to obtain the mean of ten-hand motions. Standard deviation is a measure of how extensively values are different from the average value of the group. We presented two different results in training, testing and compared them by finding the best tool to detect mini gestures. Training accuracy is achieved by implementing a model on the training data and obtaining accuracy of the algorithm, whereas testing accuracy is an accuracy for the testing data. The three provided algorithms (WT, EMD and CCN) in this paper are characterised by different measures. The total time execution for WT in four stages (Data input, Pre-processing, Image segmentation, Feature extraction) is less than the total time execution of EMD.

Figure 3 and figure 4 shows the signal extracted features using IMF method for 10 different gestures in WT and EMD techniques respectively. IMF function is used or applied under two conditions: First condition is for the entire data, the number of extrema and the number of zero crossings should also equal or vary at most by one. The second condition is the mean value of the envelope explained by the local maxima or the envelope clarified by the local minima is zero [17]. The extracted features are each assigned a class and fed to ANN for training. 


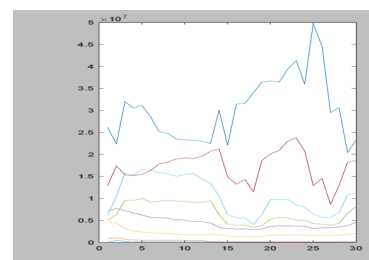

a) Sweep motion

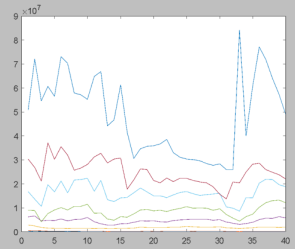

d) Squeeze motion

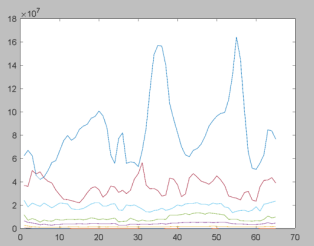

g) Rub motion

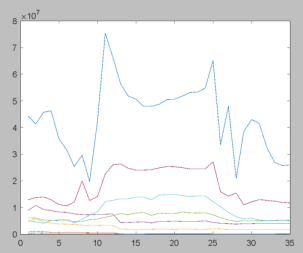

b) Shrink motion

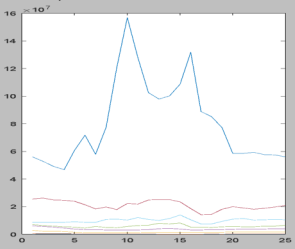

e) 2 Fingers Shrink

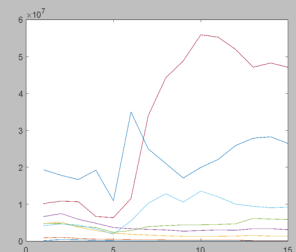

h) Click motion

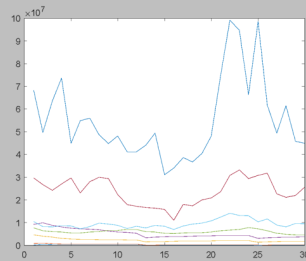

c) Circular motion

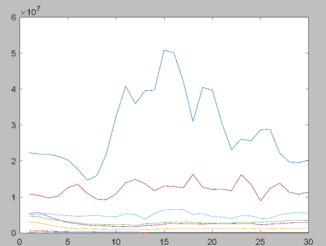

f) Back and Forth

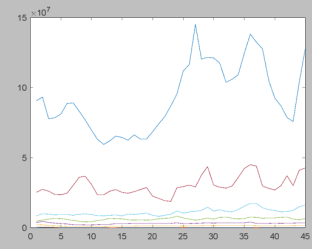

i) Dance motion

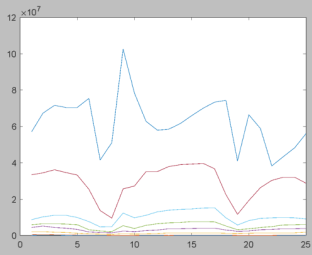

j) Pinch motion

Figure 3. IMF for 10 different motions using WT

The ten graphs above describe the axis $\mathrm{X}$ in microsecond, axis $\mathrm{Y}$ in frequency with 8 signals of IMF (levels). The speed of motion starts from 0 microsecond till the end of time with a stable signal rate. As shown in figure 3a, the blue signal has a slight change whereas other signals are steadier. All figures show dramatic increase in blue, red and light blue signals while other signals are steady

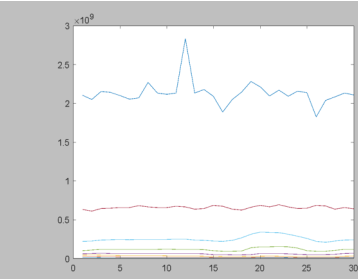

a) Sweep motion

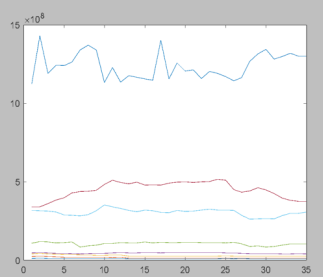

b) Shrink motion

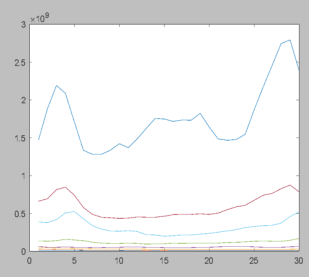

c) Circular motion 


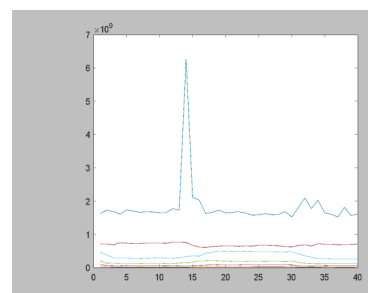

d) Squeeze motion

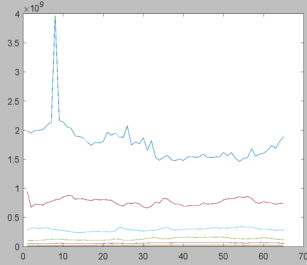

g) Rub motion

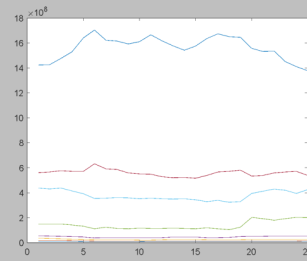

e) 2 Fingers Shrink

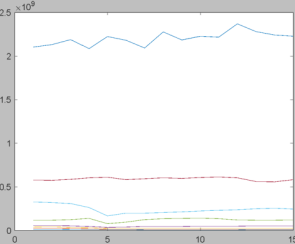

h) Click motion

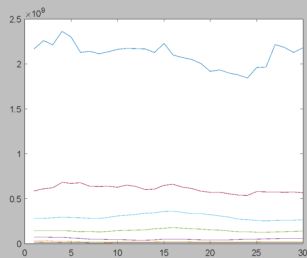

f) Back and Forth

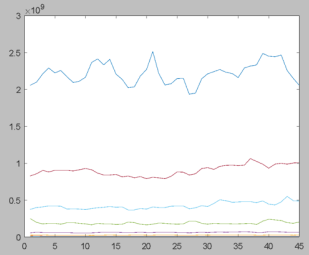

i) Dance motion

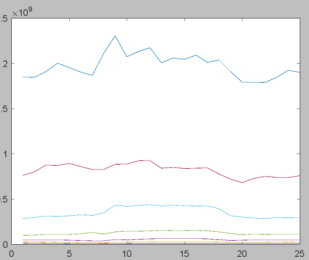

j) Pinch motion

Figure 4. IMF for 10 different motions using EMD

Table 1 shows the summary of values acquired against the parameters when being in training mode. The total time execution for WT, EMD and CNN in training. The execution time of WT is less than the total time execution of EMD and CNN. The accuracy results of CNN exceeded WT and EMD by acquiring the highest value. The value of sensitivity in CNN is higher than WT and EMD. Specificity in WT reached the top while EMD is less than WT and CNN. The PPV and NPV of WT is lower than EMD and CNN. CNN had the best value in LR+ and LR- unlike WT and EMD. The AUC is 0.97 for WT, 0.99 for EMD, and 1 for CNN respectively. The last factor is RMS, the value of EMD and CNN slightly decreased while WT has a high value in RMS.

Table 1. Comparison between WT, EMD and CNN for Training

\begin{tabular}{|c|c|c|c|}
\hline & WT & EMD & CNN \\
\hline Exe Time \pm SD (min) & $5.794 \pm 0.895$ & $9.869 \pm 1.778$ & $713.694 \pm 122.640$ \\
\hline Accuracy \pm SD & $0.400 \pm 0.072$ & $0.618 \pm 0.120$ & $1 \pm 0$ \\
\hline Sensitivity \pm SD & $0.923 \pm 0.041$ & $0.983 \pm 0.008$ & $1 \pm 0$ \\
\hline Specificity \pm SD & $7.756 \pm 8.07$ & $0.738 \pm 0.231$ & $1 \pm 0$ \\
\hline Positive Predictive Value (PPV) & $0.557 \pm 0.138$ & $0.778 \pm 0.079$ & $1 \pm 0$ \\
\hline Negative Predictive Value (NPV) & $0.935 \pm 0.010$ & $0.963 \pm 0.011$ & $1 \pm 0$ \\
\hline Positive Likelihood (LR + ) & $18.871 \pm 22.717$ & $54.628 \pm 64.926$ & $1 \pm 0$ \\
\hline Negative Likelihood (LR-) & $0.713 \pm 0.119$ & $0.396 \pm 0.123$ & $1 \pm 0$ \\
\hline RMS \pm SD & $2.420 \pm 1.452$ & $0.850 \pm 0.128$ & $1 \pm 0$ \\
\hline AUC \pm SD & $0.984 \pm 0.021$ & $0.994 \pm 0.009$ & $1 \pm 0$ \\
\hline
\end{tabular}

The parameter values of $\mathrm{CNN}$ is constant for all categories. Its execution time is approximate 714 minutes which is huge and not preferred in experiments. In this study, only ten pictures of different hand movement were involved to train the system. If the input would consist of more images, the execution time would increase proportionally which would be not ideal for any experiment in terms of time constraint. Positive Likelihood (LR+) of EMD is higher as it refers to being 
more specific towards accuracy as compared to WT and CNN algorithms. Overall, CNN has the highest value in all parameters testing when training was done except for execution time and Positive Likelihood (LR+). Thus, we can compromise on few factors to get best results when training of the system is being done.

Table 2 compares the three algorithms performance while they were being tested for this study. CNN had the total execution time, higher than WT and EMD. In accuracy factor, WT achieved a value which is lower than EMD and CNN. The accuracy results of CNN surpassed WT and EMD with a high value. The value of sensitivity in CNN is committed than that of WT and EMD. Specificity in WT is higher than EMD and CNN. EMD and CNN had higher values in PPV and NPV comparing with WT which had lower value. In LR+ and LR-, the value of CNN is higher than WT and EMD. The value of RMS for EMD algorithm significantly declined while WT has a higher value.

Table 2. Comparison between WT, EMD and CNN for Testing

\begin{tabular}{|c|c|c|c|}
\hline & WT & EMD & CNN \\
\hline Exe Time \pm SD (min) & $0.204 \pm 0.030$ & $0.192 \pm 0.060$ & $713.694 \pm 122.640$ \\
\hline Accuracy \pm SD & $0.3947 \pm 0.069$ & $0.620 \pm 0.133$ & $0.971 \pm 0.007$ \\
\hline Sensitivity \pm SD & $0.331 \pm 0.225$ & $0.554 \pm 0.245$ & $1 \pm 0$ \\
\hline Specificity \pm SD & $0.936 \pm 0.038$ & $0.733 \pm 0.368$ & $1 \pm 0$ \\
\hline Positive Predictive Value (PPV) & $0.673 \pm 0.416$ & $0.756 \pm 0.223$ & $1 \pm 0$ \\
\hline Negative Predictive Value (NPV) & $0.930 \pm 0.021$ & $0.9676 \pm 0.015$ & $1 \pm 0$ \\
\hline Positive Likelihood (LR+) & $9.103 \pm 8.785$ & $22.422 \pm 24.924$ & $1 \pm 0$ \\
\hline Negative Likelihood (LR-) & $0.681 \pm 0.155$ & $0.392 \pm 0.194$ & $1 \pm 0$ \\
\hline RMS \pm SD & $1.9780 \pm 0.901$ & $0.835 \pm 0.200$ & $1 \pm 0$ \\
\hline AUC \pm SD & $0.975 \pm 0.027$ & $0.992 \pm 0.017$ & $1 \pm 0$ \\
\hline
\end{tabular}

Again in the testing phase, CNN algorithm took similar time i.e. 714 minutes for executing the testing task. This is not feasible outcome as testing 10 images takes such long time; if mire images would be needed to be tested for experiments, the members of the experiment would have to wait to get results the whole time. CNN has 1 value in all parameters i.e. sensitivity, specificity, PPV, NPV, negative likelihood (LR-), RMS and AUC. In testing phase, the Positive Likelihood (LR+) has 1 value too which is least as compared to WT and EMD that recorded 9.1 and 22.4 respectively. Again, this proves that for testing too, $\mathrm{CNN}$ offers best results, but compromise on execution time needs to be ignored.

Figs. 5 and 6 are shown the Receiver Operating Characteristic ROC curve which is applied in binary classification to learn the output of a classifier. Currently, there are two strategies of ROC to be drawn for multiclasse curve, one vs. one and one vs. multi and the experiment used one vs. multi method. According to the WT and EMD graphs, the 10 classes had 10 ROC curves reached the upper left corner which are \%100 True Positive Rate (Sensitivity) and \%100 False Positive Rate (1-Specificity). The ROC curve of EMD is slightly near to the upper left corner more than WT.

The functionality of CNN is way better than WT and EMD, according to the results of ten-hand motions. WT and CNN used a low amount of memory while EMD used a large amount of memory as it also takes a long time to run the code.

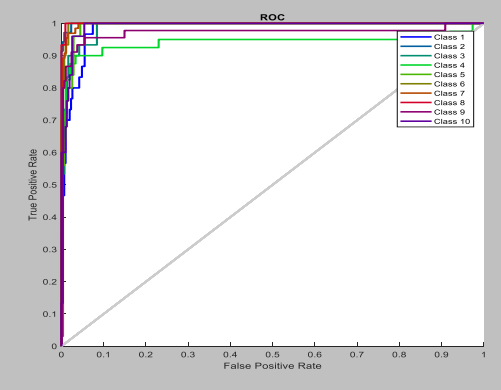

Figure 5. ROC for 10 different classes in WT

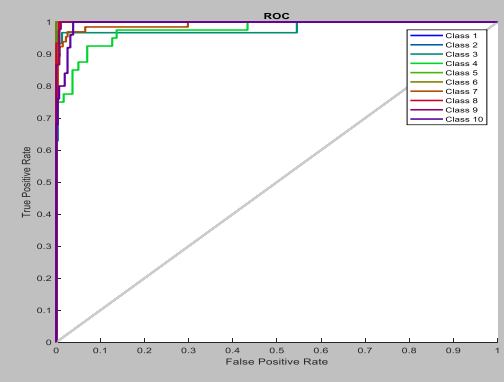

Figure 6. ROC for 10 different classes in EMD 


\section{CONCLUSIONS AND FUTURE WORK}

Gesture tracking in HCI world is the first stage to generate natural HCI system. It can be applied in applications such as gesture recognition. Segmentation of hand, detection of hand parts, and tracking of hands are the most significant parts to deal with in gesture recognition studies. The most frequently used in gesture recognition system is hand gestures. Most of the cameras can detect hand motions in the 2-D surface. There are three main phases which must be applied to hand gesture systems. Firstly, an image detects via a camera. Second phase, the system will receive the image to track it by using any image processing tools such as WT or EMD. Final stage, the data will be classified using ANN and CNN. In this study, a system has been created for hand motion detection using WT and EMD to extract the features while the classification is done using ANN and CNN. The results of this experiment show the advantages and disadvantages of each method. CNN is more accurate than WT and EMD. In future work, we will extend the number of motions using 3D horoscopic imaging system.

\section{ACKNOWLEDGEMENTS}

The first author would like to show his appreciation in terms of the financial support received from the Ministry of Higher Education in Saudi Arabia.

\section{REFERENCES}

[1] Rao, M. V. G., Kumar, P. R., Prasad, A. M., "Implementation of real time image processing system with FPGA and DSP," 2016 International Conference on Microelectronics, Computing and Communications (MicroCom) (2016).

[2] Zaitoun, N. M., Aqel, M. J., "Survey on Image Segmentation Techniques," Proceedings. Computer Science 65, 797$806(2015)$

[3] Dhanachandra, N., Chanu, Y. J., "A Survey on Image Segmentation Methods using Clustering Techniques," European Journal of Engineering Research and Science 2(1), 15 (2017).

[4] Szeliski, R., "Computer vision: algorithms and applications" (2010).

[5] Gevers, T., Voortman, S., Aldershoff, F., "Color feature detection and classification by learning," IEEE International Conference on Image Processing 2005 (2005).

[6] Murthy, G. R., Jadon, R. S., "A review of vision based hand gestures recognition," International Journal of Information Technology and Knowledge Management 2, 405-410 (2009).

[7] Pradipa, R., Kavitha, S. "Hand Gesture Recognition - Analysis of Various Techniques, Methods and Their Algorithms," International Journal of Innovative Research in Science, Engineering and Technology 2003-2010 (2014).

[8] Manresa, C., Varona, J., Mas, R., Perales, F. J., "Hand Tracking and Gesture Recognition for Human-Computer Interaction," ELCVIA Electronic Letters on Computer Vision and Image Analysis 5(3), 96 (2005).

[9] Pang, S., del Coz, J., Yu, Z., Luaces, O., Díez, J., "Deep Learning to Frame Objects for Visual Target Tracking," Engineering Applications of Artificial Intelligence. 65, 406-420 (2017).

[10]T. Bouchrika, O. Jemai, M. Zaied and C. Amar, "Rapid and efficient hand gestures recognizer based on classes discriminator wavelet networks", Multimedia Tools and Applications, vol. 77, no. 5, pp. 5995-6016, 2017.

[11] T. Bouchrika, M. Zaied, O. Jemai and C. Ben Amar, "Neural solutions to interact with computers by hand gesture recognition", Multimedia Tools and Applications, vol. 72, no. 3, pp. 2949-2975, 2013.

[12] Daubechies, I., “Ten lectures on wavelets. SIAM, Philadelphia,” PA. (1992).

[13]Huang, N., Shen, Z., Long, S., Wu, M., Shih, H., Zheng, Q., Yen, N., Tung, C., Liu, H., "The Empirical Mode Decomposition and the Hilbert Spectrum for Nonlinear and Non-stationary Time Series Analysis," Proceedings of the Royal Society A: Mathematical, Physical and Engineering Sciences. 454, 903-995 (1998).

[14]Lambert, M., Engroff, A., Dyer, M., Byer, B., "Empirical Mode Decomposition," https://www.clear.rice.edu/elec301/Projects02/empiricalMode/

[15] Aleksander, I., Taylor, J., “Artificial neural networks," 2. North-Holland, Amsterdam (1992).

[16]Fritzke, B., "Growing Cell Structures - a Self-organizing Network in k-Dimensions," Artificial Neural Networks. 1051-1056 (1992).

[17] Junsheng, C., Dejie, Y., Yu, Y., "Research on the Intrinsic Mode Function (IMF) Criterion in EMD Method. Mechanical Systems and Signal Processing," 20, 817-824 (20XX). 